\title{
Set up Invariable Axiom of Force Equilibrium and Solve Problems about Transformation of Force and Gravitational Mass
}

\author{
Kexin Yao ${ }^{1}$ \\ ${ }^{1}$ Institute of Mechanical Engineering of Shaanxi Province, Xi'an Metering Institution, Xi'an, P. R. China \\ Correspondence: Kexin Yao, Institute of Mechanical Engineering of Shaanxi Province, Room 1-7-1, Staff \\ Building, Xian Metering Institution, No. 12, Laodong South Road, Xi'an, 710068, P. R. China. Tel: \\ 86-134-8462-6424. E-mail: yayydwpq@163.com
}

Received: August 29, 2012 Accepted: October 10, 2012 Online Published: January 9, 2013

doi:10.5539/apr.v5n1p72

URL: http://dx.doi.org/10.5539/apr.v5n1p72

\begin{abstract}
Invariable axiom of force equilibrium is set up according to the common knowledge that force equilibrium is independent of observers. The transformation formulas of force in different inertial system are derived and the validity of these transformation formulas is affirmed via deducing the electric-field distribution of high speed moving charge. And through deduction, conclude that the gravitational mass of an object is not equal to its inertial mass.
\end{abstract}

Keywords: force equilibrium, electric field, special relativity theory, transformation, gravitational mass

\section{Introduction}

Special relativity theory elucidates the transformation relation of the length, time and mass between two inertial systems, but does not specify the transformation relation of acting force. This paper determines the transformation relation of forces according to the invariable axiom of force equilibrium, a new theory proposed by the author, making the length, time and mass become the fundamental transformation relations of the special relativity theory, and deducing that gravitational mass has nothing to do with its moving speed according to the transformation relation of force.

\section{Invariable Axiom of Force Equilibrium}

When a $1 \mathrm{~kg}$ object rests on a spring balance, the pointer of the spring balance will stop at the position of $1 \mathrm{~kg}$ scale. For the person relatively stationary to the spring balance, this scale indicates $1 \mathrm{~kg}$, and for other persons in motioning state will also see that the spring balance indicates $1 \mathrm{~kg}$. It means that gravity and spring force maintain balance at $1 \mathrm{~kg}$ scale position and this balanced state does not change in different inertial systems. This objective fact is a common knowledge to all. Naturally we can further deduce that a set of various forces maintaining a balanced state, which will not change in any inertial system. This acknowledged principle is called invariable axiom of force equilibrium.

\section{Variation of Force in Different Inertial Systems}

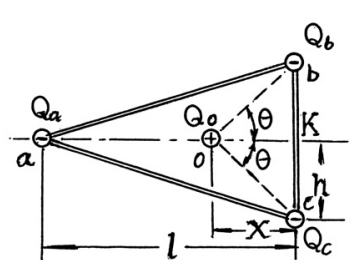

(a)

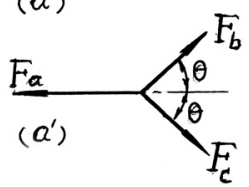

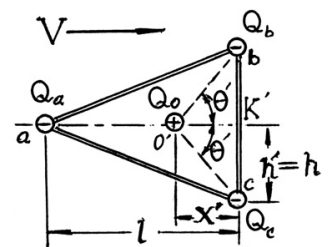

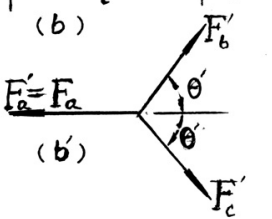

Figure 1. Motion of Equilibrium Force and Force Conversion 
In Figure 1, (a) at upper left represents an isosceles triangle support $\mathrm{abc}(\mathrm{ab}=\mathrm{ac})$ and three negative charges $-Q_{a}$, $-Q_{b},-Q_{c}$ were placed on the three vertexes of the support. We made $-Q_{b}=Q_{c}$. It is already known that the height of triangle ak $\perp \mathrm{bc}$, placing a positive charge $+Q_{0}$ at point $\mathrm{O}$ on ak, the gravitation acted upon $-Q_{0}$ by $-Q_{a},-Q_{b}$, $-Q_{c}$ are $F_{a}, F_{b}, F_{c}$ respectively. As far as scalar quantity is concerned, $F_{b}=F_{c}$, the resultant force of $F_{a}, F_{b}, F_{c}$ is as shown in $\left(a^{\prime}\right)$ in Figure 1.

These three forces maintain a balanced state, there is

$$
F_{b} \cos \theta+F_{c} \cos \theta=F_{a}
$$

As $F_{b}=F_{c}$ in value, making $F_{b}=F_{c}=F$, thus there is

$$
2 F \cos \theta=F_{a}
$$

If abc support and $-Q_{a},-Q_{b},-Q_{c}$ as well as $Q_{o}$ that maintain force equilibrium motion along ak at speed $v$, according to the special relativity theory, the length ak in direction of motion is shorted to $a^{\prime} k^{\prime}$ as shown in $(b)$ in Figure $1 . l$ and $l^{\prime}$ respectively represent ak and $a^{\prime} k^{\prime}$ and $x$ and $x^{\prime}$ respectively represent $a k$ and $a k$, according to the special relativity theory, there is $l^{\prime}=l \sqrt{1-v^{2} / c^{2}}$ ( $\mathrm{c}$ in the formula is velocity of light); $x^{\prime}=x \sqrt{1-v^{2} / c^{2}}$. The length of vertical direction of motion remains unchanged, thus there is $b^{\prime} c^{\prime}=b c, b^{\prime} k^{\prime}=b k=\mathrm{h}$ and forces $F_{a}, F_{b}, F_{c}$ accordingly change into $F_{a}, F_{b}, F_{c}$. The composition of forces is $\theta^{\prime}=\angle b^{\prime} o^{\prime} k^{\prime}, F_{b}=F_{c}$ as shown in ( $\left.b^{\prime}\right)$ in Figure 1 .

According to invariable axiom of force equilibrium, after four electric charges with interaction forces maintaining balance as shown in (a) in Figure 1 is transferred to the new inertial system as shown in (b) in Figure 1 , the interaction forces still maintain balance, namely the resultant force of $F_{a}, F_{b}, F_{c}$ is zero, therefore there is

$$
2 F_{b}^{\prime} \cos \theta^{\prime}=F_{a}^{\prime}
$$

To correspond with the previous formula and make $F^{\prime}=F_{b}$, then the above formula is revised to

$$
2 F^{\prime} \cos \theta^{\prime}=F_{a}^{\prime}
$$

Suppose the conversion ratio of $F_{a}{ }^{\prime}$ and $F_{a}$ is $\mathrm{k}$ (the specific value of $\mathrm{k}$ is to be determined), namely $F_{a}{ }^{\prime}=k F_{a}$ By comparing with $2 F \cos \theta=F_{a}$, it is known that

$$
F^{\prime} \cos \theta^{\prime}=k F \cos \theta
$$

From (a) and (b) in Figure 1, it is known that

$$
\cos \theta=x / \sqrt{x^{2}+h^{2}} ; \cos \theta^{\prime}=x^{\prime} / \sqrt{x^{\prime 2}+h^{2}}
$$

Substitute $x^{\prime}=x \sqrt{1-v^{2} / c^{2}}$ into $\cos \theta^{\prime}$, there is

$$
\cos \theta^{\prime}=\sqrt{\frac{x^{2}\left(1-v^{2} / c^{2}\right)}{x^{2}\left(1-v^{2} / c^{2}\right)+h^{2}}}
$$

Substitute the above $\cos \theta$ and $\cos \theta^{\prime}$ relational expressions into $F^{\prime}=k F \cos \theta / \cos \theta^{\prime}$, there is

$$
F^{\prime}=k F \sqrt{\frac{1-\left[x^{2} /\left(x^{2}+h^{2}\right)\right] v^{2} / c^{2}}{1-v^{2} / c^{2}}}=k F \sqrt{\frac{1-v^{2} \cos ^{2} \theta / c^{2}}{1-v^{2} / c^{2}}}
$$




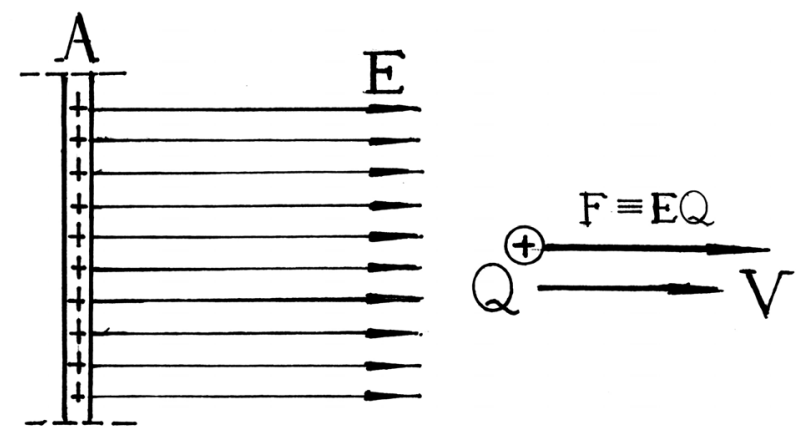

Figure 2. Force motioning parallel to $\mathrm{V}$ is irrelevant to $\mathrm{V}$

Here we use a special example to analyze ratio $\mathrm{k}$.

In Figure 2, $A$ is an infinite flat plate with electric charge, which produces a uniform electric field with field intensity of $E$. the force acting on the positive electric charge is $F=E Q ; Q$ motions along the line of electric force at speed $v$, as charged flat plate $A$ is vertical to $v, A$ does not shrink and the field intensity remains unchanged $E^{\prime}=E . F^{\prime}=E^{\prime} Q=E Q=F, K=F^{\prime} / F=1$. Substitute $K=1$ into the above formula, there is

$$
F^{\prime}=F \sqrt{\frac{1-v^{2} \cos ^{2} \theta / c^{2}}{1-v^{2} / c^{2}}}
$$

$\because F^{\prime} \cos \theta^{\prime}=k F \cos \theta=F \cos \theta$

$\therefore \cos \theta^{\prime}=\cos \theta F / F^{\prime}=\cos \theta \sqrt{\frac{1-v^{2} / c^{2}}{1-v^{2} \cos ^{2} \theta / c^{2}}}$

$$
\theta^{\prime}=\cos ^{-1} \cos \theta \sqrt{\frac{1-v^{2} / c^{2}}{1-v^{2} \cos ^{2} \theta / c^{2}}}
$$

The Formulas (1) and (2) express the force $F$ in stationary state and included angle $\theta$ formed by force $F$ and horizontal direction. After motioning at speed $v$ in horizontal direction, force $F$ produced force $F^{\prime}$ and included angle $\theta^{\prime}$.

\section{Electric Field Distribution of Speed Charged Particle}

Electrodynamics (Electrodynamics Teaching and Research Group of Tsinghua University, 1964) derived the electric field distribution formula of high speed charged particle as shown in Formula (3)

$$
E^{\prime}=k \frac{1-v^{2} / c^{2}}{\left(1-v^{2} \sin ^{2} \theta^{\prime} / c^{2}\right)^{3 / 2}} \frac{Q}{r^{\prime 2}}
$$

Where $Q$ indicates the carrying capacity of charged particle; $v$ indicates motion speed of $Q ; \mathrm{r}^{\prime}$ indicates the distance from point $E^{\prime}$ to $Q ; \theta^{\prime}$ indicates the included angle between $r^{\prime}$ and $v ; k$ indicates constant quantity of electrostatic force.

We can use force conversion formula to deduce the electric field force distribution Formula (3);

If $Q$ keeps static, the electric field force acting on a charge is $F$; when $Q$ motions at speed $v$, the electric field force acting on the charge is $F^{\prime}$. As the electric field force is directly proportional to the field intensity, the electric field force conversion formula can be written as field intensity conversion formula:

$$
E^{\prime}=E \sqrt{\frac{1-v^{2} \cos ^{2} \theta / c^{2}}{1-v^{2} / c^{2}}}
$$


Where $E^{\prime}$ indicates the field intensity of $F^{\prime}$ and $E$ indicates the field intensity of $F$.

If numerator and denominator in Formula (4) multiply $\left(1-v^{2} / c^{2}\right)\left(1-\cos ^{2} \theta v^{2} / c^{2}\right)$, there is

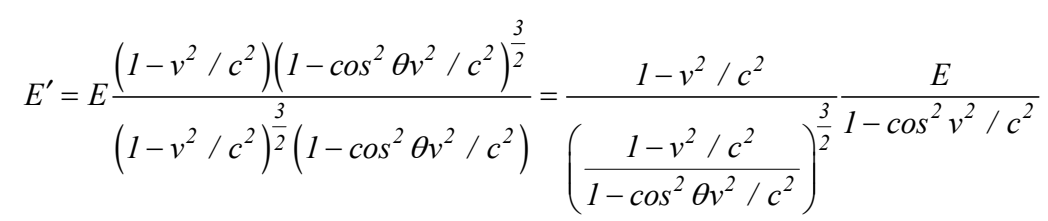

Since $1-v^{2} / c^{2}=1-\sin ^{2} \theta v^{2} / c^{2}-\cos ^{2} \theta v^{2} / c^{2}$, the above formula can be converted to

$$
E^{\prime}==\frac{1-v^{2} / c^{2}}{\left(1-\frac{\sin ^{2} \theta v^{2} / c^{2}}{1-\cos ^{2} \theta v^{2} / c^{2}}\right)^{\frac{3}{2}}} \frac{E}{1-\cos ^{2} v^{2} / c^{2}}
$$

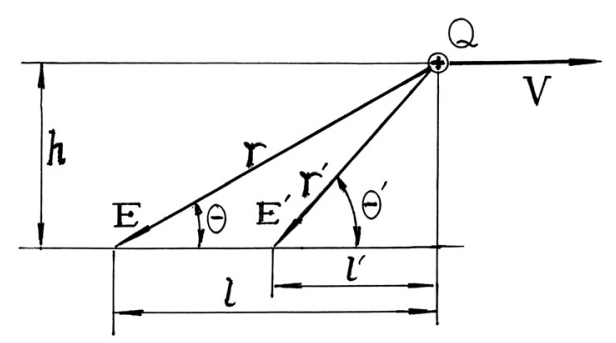

Figure 3. Deduction of Electric Field Distribution of Motional Charged Particle

Figure 3 indicates the shrinking graph of $Q$ in motioning. $r$ and $\mathrm{r}^{\prime}$ respectively indicate the change of distance before and after motion of $Q ; l$ and $l^{\prime}$ respectively indicate the change of length in $V$ direction before and after motion of $Q$. According to special relativity theory, there is $l^{\prime}=l \sqrt{1-v^{2} / c^{2}} ; \theta$ and $\theta^{\prime}$ respectively indicate the included angle between $r, r^{\prime}$ and $V$. From the figure, it is known that $r=\sqrt{l^{2}+h^{2}} ; r^{\prime}=\sqrt{l^{2}+h^{2}}$. Since $l^{\prime}=l \sqrt{1-v^{2} / c^{2}}$, there is

$$
\frac{r^{\prime}}{r}=\sqrt{\frac{l^{2}+h^{2}-l^{2} v^{2} / c^{2}}{l^{2}+h^{2}}}=\sqrt{1-\frac{l^{2}}{l^{2}+h^{2}} v^{2} / c^{2}}
$$

Since $l^{2} /\left(l^{2}+h^{2}\right)=\cos \theta$

There is

$$
r^{\prime}=r \sqrt{1-\cos ^{2} \theta v^{2} / c^{2}}
$$

From the figure it is known that $\sin \theta=h / r ; \sin \theta^{\prime}=h / r^{\prime}$, thus $\sin \theta^{\prime}=r \sin \theta / r^{\prime}$. Substitute Formula (6) into this formula, there is:

$$
\sin \theta^{\prime}=\frac{\sin \theta}{\sqrt{1-\cos ^{2} \theta v^{2} / c^{2}}}
$$

Substitute $E=k Q^{2} / r^{2}$ and Formulas (6), (7) into Formula (5), there is

$$
E^{\prime}==k \frac{1-v^{2} / c^{2}}{\left(1-\sin ^{2} \theta v^{2} / c^{2}\right)^{\frac{3}{2}}} \frac{Q}{r^{\prime 2}}
$$

This is front electric field distribution Formula (3). It shows that Formula (3) can be used to clearly express the distribution of electric field, but inconvenient to be used to calculate electric field after motion of $Q$, because $\theta^{\prime}$ and $r^{\prime}$ must be firstly calculated. But the use of electric field force conversion Formula (4) can directly acquire the answer. 


\section{Inertial Mass is not Equal to Gravitational Mass}

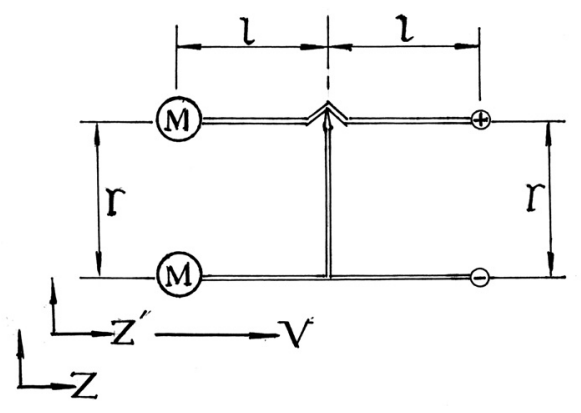

Figure 4. Universal gravitation and electric field force equilibrium are irrelevant to motion

In Figure 4, two objects with the mass of $\mathrm{M}$ and two charges of different polarity with same electric quantity in inertial system $Z^{\prime}$ constitute a force equilibrium system. The universal gravitation of two objects is $F_{M}=G M^{2} / r^{2}$ (G-constant quantity of universal gravitation); the electrostatic attraction of two charges of different polarity is $F_{Q}=k Q^{2} / r^{2}$. Under the circumstance of force equilibrium, certainly there is $F_{M}=F_{Q}$.

Now inertial system $Z^{\prime}$, relative to inertial system $Z$, motions at speed $v$. According to the invariable axiom of force equilibrium, the force equilibrium state of inertial system $Z^{\prime}$ in Figure 4 is still balanced for inertial system Z. But according to the force conversion Formula (1) and for Z, the gravitation between two Qs in inertial system $Z^{\prime}$ should be:

$$
F_{Q}^{\prime}=F \sqrt{\frac{1-v^{2} \cos ^{2} \theta / c^{2}}{1-v^{2} / c^{2}}}=\frac{k Q^{2}}{r^{2} \sqrt{1-v^{2} / c^{2}}}=\frac{F_{Q}}{\sqrt{1-v^{2} / c^{2}}}
$$

In above formula, since $r \perp v$, there is $\cos \theta=0 \quad r^{\prime}=r$

According to special relativity theory, when $\mathrm{M}$ motions at speed $v$, M should convert to $M^{\prime}=M / \sqrt{1-v^{2} / c^{2}}$, thus for $\mathrm{Z}$, the universal gravitation between two Ms in inertial system $\mathrm{Z}^{\prime}$ should be:

$$
F_{M}^{\prime}=G \frac{M^{\prime 2}}{r^{2}}=\frac{G M^{2}}{r^{2}\left(1-v^{2} / c^{2}\right)}=\frac{F_{M}}{1-v^{2} / c^{2}}
$$

Since $F_{Q}=F_{M}$, consequentially there is $F_{\mathrm{M}}^{\prime}>F_{\mathrm{Q}}^{\prime}$. From inertial system $\mathrm{Z}$, it can be found that the gravitation between two Ms is greater than that between two Qs. When force loses balance, two Ms will collide, which obviously does not comply with invariable axiom of force equilibrium and is impossible to occur. This indicates that the above deduction is wrong.

If we use gravitational field to analyze the universal gravitation, we will also obtain a result of disequilibrium. Because by inferring to the electric field distribution Formula (3) of motional charged particle, when the object is motioning, the field intensity in its vertical motion direction will convert to $M^{\prime} / \sqrt{1-v^{2} / c^{2}}$ and $M^{\prime}=M / \sqrt{1-v^{2} / c^{2}}$, the universal gravitation of two objects should be the following

$$
F_{M}^{\prime}=G M^{\prime 2} / r^{2} \sqrt{1-v^{2} / c^{2}}=F_{M} /\left(1-v^{2} / c^{2}\right)^{3 / 2}
$$

also there is $F_{\mathrm{M}}^{\prime}>F_{\mathrm{Q}}^{\prime}$.

How to correct the above improper deduction? It can be found that $F_{Q}{ }^{\prime}=F_{Q} / \sqrt{1-v^{2} / c^{2}}$ is derived from force conversion Formula (3), which conforms to the invariable axiom of force equilibrium, thus it should not be revised. Therefore, we have to make amendment from the aspect of deduction of the universal gravitation. The analysis shows that it comes possible only by taking $\mathrm{M}$ as $Q$ that having nothing to do with motional speed. Under this circumstance, the distribution of gravitational field is completely similar to the electric field and the change of field intensity of motional $\mathrm{M}$ and $Q$ is completely similar, there certainly is $F_{\mathrm{M}}^{\prime}=F_{\mathrm{Q}}^{\prime}$. Therefore, a rational deduction is that the mass of an object has duality. In terms of the universal gravitation, it expresses as 
gravitational mass $M_{0}$, which is constantly equal to the rest mass of an object having nothing to do with the motion of an object. The expression of the universal gravitation should be as follows:

$$
F_{M_{0}}=k M_{01} M_{02} / r^{2}
$$

In terms of quantity and energy of motion, it expresses as inertial mass and its value has nothing to do with motional speed $v$. According to special relativity theory, there is inertial mass: $M=M_{0} / \sqrt{1-v^{2} / c^{2}}$.

Obviously, $\mathrm{M} \neq \mathrm{M}_{0}$ Strictly speaking, inertial mass is not equal to gravitational mass and the equivalence principle of principle of relativity is untenable when an object moves at high speed. Of course, when the movement speed of an object is not high, it can be deemed that $\mathrm{M}=\mathrm{M}_{0}$ and equivalence principle is tenable. But when the movement speed of an object approaches the velocity of light, for example, $v=0.99 \mathrm{c}$, then there is $\mathrm{M}>7.2 \mathrm{M}_{0}$, and inertial mass clearly differs from gravitational mass. It is deemed that this difference is reflected on the movement track of particles under the effect of gravity. Up to now there is no experiment to prove the existence of this difference. It is suggested to testify the existence of this difference by relevant experts.

\section{Conclusion}

According to the invariable axiom of force equilibrium, the above contents, combining the length transformation of the special relativity theory, analyzes and determines the transformation relation between two inertial systems. The transformation formula derives correct result by deducing the electric field distribution of high-speed charged particles. This deduction verifies the transformation formula of force in this paper is correct. Based on this new theory, this paper derives the conclusion that gravitational mass of an object has nothing to do with its motioning speed. If this conclusion can be proved by experiments, it will have a theoretic significance for physics.

\section{References}

Electrodynamics Teaching and Research Group of Tsinghua University. (1964). Bingjing, Tsinghua University, p.208

Guo, S. H. (2010). Electrodynamics. China: China Higher Education Press.

Guo, Y. Z., \& Li, Z. X. (2011). Fundamentals of Applied Mechanics. China: China Higher Education Press

Zhang, Z. H. (2005). Fundamentals of Principle of Relativity. Beijing: TsinghuaUniversity Press. 